\title{
High polymorphism in big defensin gene expression reveals presence-absence gene variability (PAV) in the oyster Crassostrea gigas
}

\author{
Rosa Rafael D. ${ }^{1,}$, , Alonso Pascal ${ }^{1}$, Santini Adrien ${ }^{1}$, Vergnes Agnes ${ }^{1}$, Bachere Evelyne ${ }^{1}$ \\ ${ }^{1}$ Ifremer, CNRS, Université Montpellier 2, IRD, and Université Montpellier 1 (UMR5119 - Ecology of \\ Coastal Marine Systems), Place Eugène Bataillon, CC80, 34095 Montpellier, France \\ * Corresponding author: Rafael D. Rosa, tel.: +55 4837216163 ; Fax: +55 4837215528 ; email \\ address : rafael.d.rosa@ufsc.br
}

\begin{abstract}
:
We report here the first evidence in an invertebrate, the oyster Crassostrea gigas, of a phenomenon of Presence-Absence Variation (PAV) affecting immune-related genes. We previously evidenced an extraordinary interindividual variability in the basal mRNA abundances of oyster immune genes including those coding for a family of antimicrobial peptides, the big defensins ( $\mathrm{Cg}$-BigDef). $\mathrm{Cg}$-BigDef is a diverse family composed of three members: Cg-BigDef1 to -3. Here, we show that besides a high polymorphism in Cg-BigDef mRNA expression, not all individual oysters express simultaneously the three $\mathrm{Cg}$-BigDefs. Moreover, in numerous individuals, no expression of $\mathrm{Cg}$-BigDefs could be detected. Further investigation at the genomic level revealed that in individuals in which the transcription of one or all $\mathrm{Cg}$-BigDefs was absent the corresponding $\mathrm{Cg}$-bigdef gene was missing. In our experiments, no correlation was found between Cg-bigdef PAV and oyster capacity to survive Vibrio infections. The discovery of P-A immune genes in oysters leads to reconsider the role that plays the immune system in the individual adaptation to survive environmental, biotic and abiotic stresses.
\end{abstract}

Highlights

$C g$-BigDefs display a high interindividual polymorphism in basal mRNA expression. - All three $\mathrm{Cg}$ BigDefs are not simultaneously expressed in a single oyster. Lack of Cg-BigDef mRNA expression is likely due to the absence of the corresponding gene. First report in an invertebrate of PresenceAbsence Variation affecting immune genes.

Keywords : mollusk bivalve, antimicrobial peptide, polymorphism, gene copy number variation, highthroughput microfluidic RT-qPCR, Vibrio infection 


\section{Introduction}

Understanding the mechanisms of resistance or susceptibility to infections in the oyster Crassostrea gigas should contribute to identify the causes of mass mortalities that affect this marine mollusk species worldwide. In this context of multifactorial disease, significant progresses were made in characterizing the oyster immune system and responses to biotic and abiotic environment factors. Particular attention has been paid to the mechanisms involved in the capability of the oyster to overcome infections (de Lorgeril et al., 2011; Schmitt et al., 2012) but also to the genetic bases that govern oyster survival (Rosa et al., 2012).

High-throughput transcriptomic studies have revealed in C. gigas great individual polymorphism in the expression of many immune-related genes (Rosa et al., 2012). This supported previous evidence that $C$. gigas is a highly polymorphic and intron-rich species in terms of number of SNPs and repetitive DNA sequences (Kim et al., 2011; Saavedra and Bachère, 2006; Sauvage et al., 2007). Thus, besides sequence polymorphism, the oyster displays great polymorphism of expression that can be related to gene Copy Number Variation (CNV) in genome contents between different individuals (Rosa et al., 2012; Schmitt et al., 2013). CNV are known to be of fundamental importance for genetic variation in populations, evolution of genome complexity and evolution of genes with novel functions. Many studies have looked for associations between the copy number of genes and disease susceptibility (reviewed in Cantsilieris and White, 2013). For instance, in human, whereas extensive copy number of $\beta$-defensin antimicrobial peptide has been associated to the risk of psoriasis (Hollox et al., 2008), low copy number of $\beta$-defensin has been reported to predispose to Crohn disease (Fellermann et al., 2006).

Surprisingly, our high-throughput transcriptomic studies have also revealed numerous genes whose basal levels of expression could not be detected in some individuals (Rosa et al., 2012). Those genes were shown to be involved in immune responses such as genes encoding 
members of the big defensin antimicrobial peptide family, $C g$-BigDef (Rosa et al., 2011). Oyster big defensins ( $\mathrm{Cg}$-BigDef) have been shown to be a diverse antimicrobial peptide family composed of three members ( $C g$-BigDef1, $C g$-BigDef2 and $C g$-BigDef3) that are encoded by distinct genomic sequences (Rosa et al., 2011). An interesting particularity of the oyster $\mathrm{Cg}$-BigDef family is that their members follow different patterns of gene regulation upon microbial challenge. Indeed, whereas $C g$-BigDefl and $C g$-BigDef2 are up-regulated in circulating hemocytes in response to pathogenic Vibrio infection, $C g-B i g D e f 3$ is constitutively expressed and non-regulated (Rosa et al., 2011). In this study, an unusual variability in mRNA expression was evidenced in oyster sampling for all Cg-BigDefs. As Cg-BigDefl and 2 transcriptions are highly induced by microbial molecular patterns (Rosa et al., 2011), it was reasonable to expect that these molecules could be implicated in preventing from development infectious processes. This prompted us to further analyze such polymorphism of expression in C. gigas.

Here, a detailed analysis of $C g$-BigDef mRNA expression at individual level confirmed firstly a high interindividual variability in basal gene expression for the three $C g$-BigDef forms. In addition, we found that the three $C g$-BigDefs are not simultaneously transcribed in a single oyster. More surprisingly, in some individual oysters, basal mRNA levels were not detected for any $C g$-BigDefs. Besides, we evidenced that the absence of $C g$-BigDef transcription is likely associated to the lack of $C g$-bigdef genes, revealing the extreme form of CNV named Presence-Absence Variation (PAV). Finally, the Presence-Absence Variation of Cg-bigdefs in the oyster genome was shown to be not related to a better capacity of the oysters to survive experimental Vibrio infections.

\section{Materials and Methods}

\subsection{Oysters and experimental Vibrio infections}


Adult two-year old Crassostrea gigas oysters were purchased from a local oyster farm same individuals.

in Mèze (Gulf of Lion, France) and kept in aquaria containing filtered seawater at $15^{\circ} \mathrm{C}$ at the Aquaculture Experimental Platform of Ifremer, Palavas-Les-Flots. During two weeks, animals were fed twice with a mixture of live microalgae (Nannochloropsis oculata: $2.5 \times 10^{5}$ cells $/ \mathrm{ml}$; Tetraselmis suecica: $2.5 \times 10^{2}$ cells $/ \mathrm{ml}$ ). Following the acclimatation period, 90 oysters were individually tagged and hemolymph $(\sim 500 \mu 1$ per animal $)$ was withdrawn from the adductor muscle without causing the death of the animals. Hemocyte samples were individually collected by centrifugation $\left(1,500 \times \mathrm{g}, 15 \mathrm{~min}, 4^{\circ} \mathrm{C}\right)$, homogenized in $1 \mathrm{ml}$ of TRIzol ${ }^{\circledR}$ reagent (Invitrogen) and frozen at $-20^{\circ} \mathrm{C}$ until RNA extraction. After hemolymph collection, animals were placed in glass tanks (20 animals per tank) and allowed to recover for 8 days prior to experimental infections. Then, animals were intramuscularly injected with $5 \times 10^{8} \mathrm{CFU} / \mathrm{animal}$ of the virulent $V$. tasmaniensis LGP32 ( $=V$. splendidus LGP32). Mortalities were monitored daily and individually tagged animals that did not survive were noted and discarded. After the end of acute mortalities (96 h post-infection), stored hemolymph samples were categorized according to oyster survival (surviving versus non-surviving). The standardization of the experimental infections and the preparation of the bacterial inoculum were performed as previously described (Duperthuy et al., 2010). All experimentations were performed according to the Ifremer animal care guideline and policy.

Hemocyte samples from 73 additional oysters (naive animals) were obtained as described above, homogenized in $1 \mathrm{ml}$ of $\mathrm{TRIzol}^{\circledR}$ reagent (Invitrogen) and frozen at $-20^{\circ} \mathrm{C}$ until RNA extraction. From those, 23 oysters were randomly chosen, removed from their shells and immediately frozen in liquid nitrogen. Frozen whole-body oysters were individually ground to fine powder for further extractions of total RNA and genomic DNA in 


\subsection{Genomic DNA isolation}

Genomic DNA (gDNA) was isolated individually as previously described (Schmitt et al., 2010). Briefly, 50-100 mg of oyster powder were incubated overnight at $55^{\circ} \mathrm{C}$ in $500 \mu \mathrm{lof}$ DNA extraction buffer $(100 \mathrm{mM} \mathrm{NaCl}, 10 \mathrm{mM}$ Tris-HCl pH 8, $25 \mathrm{mM}$ EDTA pH 8, 0.5\% SDS, $0.1 \mathrm{mg} / \mathrm{ml}$ protease $\mathrm{K}$ ). Following a step of phenol/chloroform extraction, gDNA samples were precipitated by addition of cold ethanol and treated with $50 \mu \mathrm{g} / \mathrm{ml}$ RNase A (Invitrogen) for $30 \mathrm{~min}$ at $37^{\circ} \mathrm{C}$ to eliminate contaminating RNA. Quantification and quality of gDNA samples were assessed by spectrophotometry (NanoDrop ND-1000 Thermo Scientific) and $0.8 \%$ agarose gel electrophoresis, respectively.

\subsection{Total RNA extraction and cDNA synthesis}

Total RNA was extracted individually by using TRIzol ${ }^{\circledR}$ reagent (Invitrogen) according to the manufacturer's instructions. RNA samples were then treated with DNase I (Invitrogen) for $15 \mathrm{~min}$ at room temperature to eliminate contaminating genomic DNA. After DNase I inactivation $\left(10 \mathrm{~min}\right.$ at $\left.65^{\circ} \mathrm{C}\right)$, samples were precipitated with $0.3 \mathrm{M}$ sodium acetate. RNA amount and quality were assessed by spectrophotometric analysis and the integrity of total RNA was analyzed by $0.8 \%$ agarose gel electrophoresis.

Following heat denaturation $\left(70^{\circ} \mathrm{C}\right.$ for $\left.5 \mathrm{~min}\right)$, reverse transcription was performed using $250 \mathrm{ng}$ of purified total RNA with $50 \mathrm{ng} / \mu \mathrm{l}$ oligo(dT) ${ }_{12-18}$ in a $20-\mu 1$ reaction volume containing $1 \mathrm{mM}$ dNTPs, 1 unit of RNaseOUT Ribonuclease and 200 units M-MLV reverse transcriptase in reverse transcriptase buffer according to the manufacturer's instructions (Invitrogen). Attempting to increase the number of cDNA templates, a supplementary step of preamplification was performed using $1.25 \mu \mathrm{l}$ of cDNA, $1.25 \mu \mathrm{l}$ of a mixture of forward and reverse primers $\left(200 \mathrm{nM}\right.$ each) and $2.5 \mu \mathrm{l}$ of $2 \times$ TaqMan ${ }^{\circledR}$ PreAmpMaster Mix (Applied Biosystems). The specific primer pairs for each $C g$-BigDef form are listed in Table 1. PCR 
cycling conditions were as follows: polymerase activation at $95^{\circ} \mathrm{C}$ for $10 \mathrm{~min}$, followed by 14 cycles of $95^{\circ} \mathrm{C}$ for $15 \mathrm{~s}$ and $60^{\circ} \mathrm{C}$ for $4 \mathrm{~min}$. Preamplification products were diluted to $1: 5 \mathrm{in}$ TE buffer (10 mM Tris, $1 \mathrm{mM}$ EDTA, $\mathrm{pH} 7.5)$ and stored at $-20^{\circ} \mathrm{C}$.

\subsection{High-throughput RT-qPCR using 96.96 microfluidic dynamic arrays}

The relative gene expression was measured by using the high-throughput microfluidic RT-qPCR platform BioMark ${ }^{\mathrm{TM}}$ (Fluidigm) (Jang et al., 2011). The sample reaction mixtures were performed in a final volume of $5 \mu$ l containing $1.25 \mu \mathrm{l}$ of preamplified cDNA (diluted 1:5), $2.5 \mu \mathrm{l}$ of $2 \times$ TaqMan ${ }^{\circledR}$ Gene Expression Master Mix (Applied Biosystems), $0.25 \mu 1$ of $20 \times$ DNA Binding Dye Sample Loading Reagent (Fluidigm), $0.25 \mu 1$ of $20 \times$ EvaGreen (Biotium) and $0.75 \mu \mathrm{l}$ of TE buffer. Primer reaction mixtures were made in the same volume of $5 \mu \mathrm{l}$ containing $2.5 \mu \mathrm{l}$ of $2 \times$ Assay Loading Reagent (Fluidigm), $1.25 \mu \mathrm{l}$ of $20 \mu \mathrm{M}$ of forward and reverse primer mix and $1.25 \mu$ of TE buffer. Both sample and primer reaction mixtures were loaded into the dynamic array chip that was subsequently placed on the NanoFlex ${ }^{\mathrm{TM}}$ 4-IFC Controller for loading and mixing. After approximately $50 \mathrm{~min}$, the chip was transferred to the BioMark ${ }^{\mathrm{TM}}$ Real-Time PCR System.

The cycling program used consisted of 10 min at $95^{\circ} \mathrm{C}$ followed by 40 cycles of $95^{\circ} \mathrm{C}$ for $15 \mathrm{~s}$ and $1 \mathrm{~min}$ at $60^{\circ} \mathrm{C}$. Melting curves analysis was performed after completed RT-qPCR collecting fluorescence between $60-95^{\circ} \mathrm{C}$ at $0.5^{\circ} \mathrm{C}$ increments. Data were analyzed using the BioMark $^{\mathrm{TM}}$ Real-time PCR analysis software to obtain $\mathrm{Cq}$ values. Results are presented as changes in relative expression normalized with the $\mathrm{Cq}$ geometric mean value of the eukaryotic translation elongation factor 1-alpha (Cg-efl $\alpha$ : AB122066), the ribosomal protein L40 ( $\mathrm{Cg}$ rpl40: FP004478) and the 40S ribosomal protein S6 (Cg-rps6: CU686508) as endogenous reference genes (Livak and Schmittgen, 2001). Statistical significance between expression 
detection of $C g$-BigDefs and oyster capacity to survive $V$. tasmaniensis LGP32 infections was determined by ANOVA test at $p<0.05$.

\subsection{Genotyping of Cg-bigdefs}

PCR amplifications for Cg-bigdef genotyping were conducted in a 25 - $\mu$ l reaction volume using $1 \mu \mathrm{l}$ of synthesized cDNA or $30 \mathrm{ng}$ of purified gDNA as template. Primer sequences are listed in Table 1. PCR conditions were as follows: $10 \mathrm{~min}$ at $94^{\circ} \mathrm{C}$, then 30 cycles at $94^{\circ} \mathrm{C}$ for $1 \mathrm{~min}, 56^{\circ} \mathrm{C}$ for $1 \mathrm{~min}, 72^{\circ} \mathrm{C}$ for $3 \mathrm{~min}$ and a final elongation step of $72^{\circ} \mathrm{C}$ for $10 \mathrm{~min}$. PCR products were analyzed by electrophoresis on $1.5 \%$ agarose gel stained with ethidium bromide. The amplification products were cloned into a pCR $^{\circledR}$-Blunt II-TOPO ${ }^{\circledR}$ cloning vector using a Zero Blunt ${ }^{\circledR} \mathrm{TOPO}^{\circledR}$ PCR cloning kit (Invitrogen). The positive recombinant clones were identified by colony PCR and were sequenced in both directions.

\subsection{Sequence data analysis}

Obtained sequences were analyzed for similarities using BLASTX at the National Center for Biotechnology Information (NCBI; http://www.ncbi.nlm.nih.gov). The multiple alignments were generated by using the ClustalW2 Multiple Alignment Program (ClustalW2; http://www.ebi.ac.uk/Tools/msa/clustalw2/). The phylogenetic analyses based on the fulllength cDNA and genomic sequences of $C$. gigas big defensins were performed using the Neighbour-Joining method with the software MEGA version 4 (Tamura et al., 2007). Bootstrap sampling was reiterated 1,000 times.

\section{Results}

3.1. Oyster big defensins display a high interindividual polymorphism in basal gene expression 
The basal expression levels of the three big defensins were first analyzed by a high-

196

197

198

199

200

201

202

203

204

205

206

207

208

209

210

211

212

213

214

215 throughput RT-qPCR device in 163 individual oysters (Fig. 1; Appendix A). Specific primers were used for each $C g$-BigDef form. The RT-qPCR analyses evidenced a high interindividual variability for the three $C g$-BigDef transcripts. The $C g$-bigdef1 and $C g$-bigdef2 forms whose expression is inducible showed lower basal expression compared to the Cg-bigdef 3 form, constitutively expressed. Figure $2 \mathrm{~A}$ shows the relative mRNA abundance of $C g$-BigDefs in ten individual oysters which are representatives of individual differences in basal gene expression of the 163 oysters analyzed in this study. Depending on individuals, differences reached up to 30, 27 and 7-fold for $C g$-BigDef1, $C g$-BigDef2 and Cg-BigDef3, respectively (Fig. 1; Fig. 2A). Besides, the three $C g$-BigDef forms were never found to be simultaneously expressed among the 163 oysters analyzed (Table 2). Interestingly, only one or two forms of $C g$-BigDefs were shown to be expressed in a same individual. $C g-B i g D e f 1$ and $C g$-BigDef2 were seen in 81 and 47 individuals, respectively, while $C g$-BigDef3 was expressed in 21 oysters only. Similar results were obtained when using different specific primer pairs for each Cg-BigDef form (Table 1).

More surprisingly, among the 163 oysters, 39 did not express any Cg-BigDefs (Table 2). This lack of expression was further confirmed by RT-qPCR analyses using two universal primer pairs common to all $C g$-BigDef sequences. As a control to these RT-qPCR analyses, the expression of three reference genes (Cg-rpl40, Cg-efla and Cg-rps6) was found to be uniform across all tested individuals (Appendix A). These results evidenced a high interindividual variability in gene expression of $C$. gigas big defensins, including cases where their basal expression is fully absent in some individuals.

\subsection{Lack of $C g$-BigDef expression is likely associated to the absence of encoding genes in} the oyster genome 
In light of the degree of polymorphism in gene expression observed for $C g$-BigDefs, we

221

222

223

224

further investigated the relationships between the expression and presence of the three $\mathrm{Cg}$ bigdef genes in 23 individuals from which we have extracted both mRNA and gDNA.

Detection of transcripts was performed by RT-qPCR and detection of corresponding genes by conventional PCR with specific primer pairs for each form (Table 1). The expression profiles obtained for these 23 individuals confirmed the high variability in basal expression levels observed above by high-throughput RT-qPCR analyses (Fig. 2A; Appendix A). Figure 2B shows the amplification of $C g$-bigdef genes in ten individual oysters which are representatives of the 23 oysters analyzed. Interestingly, genes encoding a given $\mathrm{Cg}$-BigDef were only amplified in individuals expressing the corresponding form at basal levels (Fig. 2B). The accuracy of these results was further confirmed by using an additional primer pair specific for the different $C g$-bigdefs and two universal primer pairs (common to all forms). Besides, as a control of the integrity of extracted gDNA samples, $C g$-efl $\alpha$ gene was successfully amplified in all oysters analyzed (Fig. 2B).

\section{3. $C g$-BigDef forms are likely encoded by three distinct genes}

We performed an exhaustive gDNA and cDNA cloning and sequencing of the $\mathrm{Cg}$ bigdefs from four individuals expressing one or two forms, first to ensure the authenticity of the PCR amplifications but also to gain insights into the genomic organization of the big defensin family. We sampled one individual that transcribed simultaneously $C g$-BigDefl and -2 as well as one individual that was shown to transcribe both $C g$-BigDefl and -3 . Two additional individuals expressing only $C g$-BigDef2 or -3 were also sampled. Both gene and cDNA sequences were obtained from these four individuals where the Cg-BigDefs were detected at the level of transcripts and genes. The obtained sequences were deposited in GenBank under the accession numbers JN251121 to JN251132. 
Multiple alignments of the $C g$-BigDef gDNA and cDNA sequences obtained in single individuals confirmed that each oyster big defensin form is transcribed by a separated gene. Indeed, whereas $C g$-bigdefl and $C g$-bigdef2 genes included two exons interrupted by a single intron, an additional intron and exon were observed in $C g$-bigdef 3 upstream the first exon common to the other Cg-bigdef genes (Fig. 3). Interestingly, in one individual in which both Cg-BigDef1 and -2 were expressed, two different Cg-bigdef1 sequences were identified in addition to the $C g$-bigdef2 gene (Fig. 4). In this oyster, the $C g$-bigdef1 genes differed in the length of the intron sequence and by the presence of two nucleotide substitutions (Fig. 4).

Moreover, for the other individuals analyzed, single nucleotide polymorphisms (SNP) were found between transcripts and genes, which was evocative of two different alleles of a same $C g$-bigdef gene. Thus, each Cg-bigdef would correspond rather to a distinct gene than to 256 alleles from a same $C g$-bigdef locus. Sequence analysis revealed that, for each Cg-bigdef gene, the length of exons was well conserved among individuals whereas the introns lengths were variable. According to individuals, SNP were identified in both intron and exon sequences for a same Cg-bigdef gene. Additionally, introns from all Cg-bigdef genes included some microsatellite sequences 261 (such as CTAT, CT and CA) and the canonical GT/AG splice site junctions (Fig. 3). The intron sequences of $\mathrm{Cg}$-bigdef 3 appeared to differ considerably in both length and nucleotide composition from those of Cg-bigdef1 and Cg-bigdef2 genes (Fig. 3).

A phylogenetic tree was built with the nucleotide sequence of six Cg-bigdef gene sequences obtained here and with $C g$-bigdef gene sequences previously deposited in GenBank 266 (Rosa et al., 2011). In this tree, Cg-bigdef1 and Cg-bigdef2 genes clustered in a same clade distinct from the $C g$-bigdef3 gene (Fig. 5). An additional phylogenetic tree constructed from cDNA sequence data confirmed that $C g$-BigDef1 and $C g$-BigDef2 forms are more related to 
analyses strongly suggest that $C g$-BigDefs constitute a multigenic family of antimicrobial peptides in C. gigas oysters.

\subsection{Presence-absence variation (PAV) in $\mathrm{Cg}$-BigDef gene expression is not associated to} oyster capacity to survive virulent Vibrio infections

In order to investigate whether the presence or absence of $C g$-BigDefs was associated to an inherent capacity of oysters to survive infections, we have quantified the mRNA levels of all three $C g$-BigDefs (Cg-BigDef1, $C g$-BigDef2 and $C g$-BigDef3) in 90 individual animals before a challenge with an oyster pathogen. By using a nonlethal sampling method prior to the experimental infections (Rosa et al., 2012), we have assessed the basal transcription profile of all three $C g$-BigDefs in oysters able (S) and not able (NS) to survive infections by the virulent $V$. tasmaniensis LGP32 (=V. splendidus LGP32). Three independent experimental infections were conducted and hemolymph samples from 45 oysters of each survival capacity phenotype were obtained.

The detection of $C g$-BigDef transcripts and their mRNA abundance were compared in individual oysters from both S and NS phenotypes by high-throughput microfluidic RT-qPCR analyses. Transcripts of $\mathrm{Cg}$-BigDefl were detected in 25 individuals from both $\mathrm{S}$ and NS phenotypes whereas transcripts of $C g$-BigDef2 were detected in 10 and 12 individuals from $\mathrm{S}$ and NS phenotypes, respectively (Table 3). Cg-BigDef3 transcripts were detected in 4 individuals from the S phenotype and in 2 from the NS phenotype. For the S phenotype, 27 and 6 individuals expressed, respectively, only one or two $C g$-BigDefs ( $C g$-BigDefl and $C g$ BigDef2 or $C g$-BigDefl and $C g$-BigDef3) and 12 oysters did not express any $C g$-BigDefs (Table 3). For the NS phenotype, 4 and 31 oysters expressed, respectively, only one or two Cg-BigDefs (Cg-BigDef1 and Cg-BigDef2 or Cg-BigDef1 and Cg-BigDef3) and 10 oysters did not express any $C g$-BigDefs. No oysters expressing simultaneously $C g$-BigDef2 and $C g$ - 
BigDef3 or all three Cg-BigDefs were found in both S and NS phenotypes (Table 3). No significant differences were found in the detection of Cg-BigDefs according to the S and NS phenotypes, namely according to the oyster capability to survive virulent Vibrio infections $(p>0.05)$. Additionally, no significant differences in basal gene expression levels of the three Cg-BigDefs were found between individuals from both survival capacity phenotypes.

\section{Discussion}

Taking advantages of the development of high-throughput genomic technologies, we have explored the genetic determinants for Crassostrea gigas capacity to survive infections at the individual level. These analyses highlighted an extraordinary interindividual polymorphism in the basal expression of genes in this species (Rosa et al., 2012). Indeed, most of the transcriptome profilings are performed with oyster sample pools, which hides great information about oyster genomic diversity and complexity. Here, high-throughput RTqPCR analyses at the individual level revealed a high variability in basal gene expression regulation of antimicrobial peptides belonging to $C g$-BigDef family. Basal mRNA amounts of both the inducibly expressed $C g$-BigDefl and $C g$-BigDef2 and the constitutively-expressed $C g$-BigDef3 forms appeared to be highly variable among individual oysters. For instance, variations up to 30 -fold were found in $C g$-BigDef1 expression according to individuals. This interindividual polymorphism in $C g$-BigDef transcription levels may reflect genetic structure variation in gene copy numbers $(\mathrm{CNV})$ as recently reported for other oyster AMPs, $C g$-Defs and $C g$-Prps (Schmitt et al., 2013).

When analyzing the heterogeneity of transcription profiles of the big defensins, we showed that no expression was detected in some oysters and that the lack of basal expression was likely associated to the absence of the corresponding encoding sequences at the genome level. Such results highlight structural variations in oyster genomes and particularly gene 
Presence-Absence variation (PAV), a phenomenon described as the extreme case of Copy Number Variation (CNV) in which a large DNA segment present in some individuals can be entirely missing in others. To our knowledge, this is the first evidence of PAV in oysters.

$$
\text { Considering that } C \text {. gigas oyster displays a high degree of DNA sequence }
$$

polymorphism and heterozygosity (Sauvage et al., 2007; Schmitt et al., 2010; Taris et al., 2008), a rigorous accuracy has been required in the choice of the primers used in this study.

Two primer pairs specific for each of the three $C g$-BigDefs have been designed as well as two primer pairs common for all three forms. The relationship between the presence-absence (PA) of transcripts and the presence-absence of corresponding encoding gene has been confirmed in 23 individuals by different PCR analyses using eight different primer pairs spread across the entire sequences. Besides, the correspondence between the different genes and their corresponding transcripts amplified has been assessed by nucleotide sequencing. Thus, the non-amplification of any $C g$-BigDef is likely due to the lack of genes instead of the existence of null alleles commonly reported in oysters (Reece et al., 2004).

Here, we have improved also knowledge about oyster big defensin diversity by the identification of novel variants for each $\mathrm{Cg}$-BigDef form. Interestingly, in single individuals, we evidenced the presence of different variants for a same $C g$-bigdef gene, suggesting the presence of different isoforms of a given $C g$-BigDef in a same oyster. Likewise, the human $D E F$ locus has been shown to be highly variable among individuals (Taudien et al., 2004). Variations in the human $D E F$ locus include the presence of different alleles for a same $D E F$ gene, and both CNV and PAV phenomena.

PAV appeared to concern all Cg-bigdefs but more particularly the constitutively expressed $C g$-bigdef3 gene that was recorded in less than $13 \%$ of the analyzed oyster population. Importantly, since we sampled animals from a same oyster hatchery, we cannot presume that the obtained frequency distribution of Cg-bigdef genes is representative of 
natural oyster populations or representative of the species. The three $C g$-BigDefs were never seen simultaneously expressed in a single oyster, probably due to the relatively limited oyster sampling size. Indeed, based on the occurrence frequency of the three Cg-bigdefs in this oyster population, the probability of one individual carrying the three loci was calculated to be less than $2 \%$. Any of the three big defensin forms were detected in $23.9 \%(39 / 163)$ of the individuals examined. A single oyster can express only one or up two Cg-BigDefs concomitantly. Conversely, in Litopenaeus shrimp species all three members of the penaeidin family (Litvan PEN2, Litvan PEN3 and Litvan PEN4) and all four members of the antilipopolysaccharide factor (ALF) family (Litvan ALF-A, Litvan ALF-B, Litvan ALF-C and Litvan ALF-D) are simultaneously transcribed in a single individual (Cuthbertson et al., 2002; O’Leary and Gross, 2006; Rosa et al., 2013). Each penaeidin member is encoded by a separated gene and the interindividual molecular diversity is mainly generated by DNA polymorphism within each PEN gene locus (O'Leary and Gross, 2006). The simultaneous expression of different members of a given antimicrobial peptide family in a single animal has been shown to improve its antimicrobial defenses (Cuthbertson et al., 2002; Rosa et al., 2013). Analyses of the $C g$-bigdef gene sequences obtained from a single individual suggest that each $C g$-BigDef form is encoded by a separate gene and not by different alleles from a same Cg-bigdef locus. Interestingly, the intron sequences of $C g$-bigdef 3 were shown to be different from those of $C g$-bigdefl and Cg-bigdef2 genes, suggesting an ancient divergence between the constitutively and the inducibly expressed forms. Likewise to $C g$-BigDefs, $C g$-Defs and $C g$-Prps are also multigenic families whose the different members are encoded by separate genes displaying distinct gene structures (Schmitt et al., 2010). Thus far, no transcription profiling of $C g$-Defs and $C g$-PRPs have been performed at the individual level but it can be assumed that PAV can also affect these effectors in oysters. 
To date, intraspecific variations in gene contents such as PAV have never been reported in oysters and any other non-model invertebrate species, probably because few genome sequences are available. Indeed, whole $C$. gigas genome has been recently sequenced from one individual (Zhang et al., 2012). It is noteworthy that we failed to evidence in the published database any full sequences of big defensin encoding genes (Zhang et al., 2012). The PAV phenomenon for big defensins or any other genes in C. gigas deserves to be fully characterized by genetic mapping or through population-scale genome sequencing.

PAV is essentially known in human and particularly in plants in which this genetic diversity is exploited in domestication programs. In human, PAV has been described for the DEFA3 gene encoding human neutrophil AMP HNP-3 (Linzmeier and Ganz, 2005). Interestingly, the occurrence of DEFA3 gene is variable depending on the human geographical populations, and it has been suspected to be implicated with differences in infectious disease susceptibility (Ballana et al., 2007). In our study, no correlation has been shown between the P-A prevalence of the big defensins and the intrinsic capacity of the oysters to survive experimental Vibrio infections. However, one can expect that P-A genes others than those genes encoding AMP could have substantial involvement on oyster fitness and health in the context of multifactorial disease that currently affects this species worldwide.

In plants, regions of the genome encompassing PAV have been shown to contain gene clusters involved in disease resistance (Zhang et al., 2014). Thus, it is assumed that PAV may play important role in generating new pathogen resistances and they are now considered as polymorphic markers for genetic studies and breeding (Wang et al., 2014; Zhang et al., 2014). Overall, the great number of P-A genes observed in plants is recognized to play important roles in individual adaptation to external pressure and environmental stresses (Haun et al., 2011; Springer et al., 2009; Swanson-Wagner et al., 2010). Indeed, such structural variations 
394

395

396

397

398

399 and abiotic stresses.

400

401

402

403

404

405

406

407

408

409

410

411

412

413

414

415

\section{Acknowledgments}

\section{References}

imply that the missing genes in some individuals are not necessary for survival but rather contribute to the genome evolution and to the interplay with the environment (Marroni et al., 2014). Undoubtedly, the first discovery of PAV that affect immune-related genes in oyster open questions about the functional consequences of such genome structural variation on the oyster immune responses but also on the individual capability to survive environmental, biotic

We are in debt with Frédérique Cerqueira, Jean-Luc Rolland and Marc Leroy for their excellent technical assistance. We are also grateful to François Bonhomme for helpful comments and Delphine Destoumieux-Garzón for critical reading of the manuscript. This study received financial support from Ifremer and CNRS. RD Rosa was supported by a doctoral founding from CNPq-Brazil. Data used in this work were partly produced through molecular genetic analysis technical facilities of the SFR "Montpellier Environnement Biodiversité" and the "Platform qPHD UM2/Montpellier GenomiX".

Ballana, E., González, J.R., Bosch, N., Estivill, X., 2007. Inter-population variability of DEFA3 gene absence: correlation with haplotype structure and population variability. BMC Genomics 8, 14.

Cantsilieris, S., White, S.J., 2013. Correlating multiallelic copy number polymorphisms with disease susceptibility. Hum. Mutat. 34, 1-13.

Cuthbertson, B.J., Shepard, E.F., Chapman, R.W., Gross, P.S., 2002. Diversity of the penaeidin antimicrobial peptides in two shrimp species. Immunogenetics 54, 442-5. 
418 de Lorgeril, J., Zenagui, R., Rosa, R.D., Piquemal, D., Bachère, E., 2011. Whole

419

420

421

422

423

424

425

426

427

428

429

430

431

432

433

434

435

436

437

438

439

440 transcriptome profiling of successful immune response to Vibrio infections in the oyster Crassostrea gigas by digital gene expression analysis. PLoS One 6, e23142.

Duperthuy, M., Binesse, J., Le Roux, F., Romestand, B., Caro, A., Got, P., Givaudan, A., Mazel, D., Bachère, E., Destoumieux-Garzón, D., 2010. The major outer membrane protein OmpU of Vibrio splendidus contributes to host antimicrobial peptide resistance and is required for virulence in the oyster Crassostrea gigas. Environ. Microbiol. 12, $951-63$.

Fellermann, K., Stange, D.E., Schaeffeler, E., Schmalzl, H., Wehkamp, J., Bevins, C.L., Reinisch, W., Teml, A., Schwab, M., Lichter, P., Radlwimmer, B., Stange, E.F., 2006. A chromosome 8 gene-cluster polymorphism with low human beta-defensin 2 gene copy number predisposes to Crohn disease of the colon. Am. J. Hum. Genet. 79, 439-48.

Haun, W.J., Hyten, D.L., Xu, W.W., Gerhardt, D.J., Albert, T.J., Richmond, T., Jeddeloh, J.A., Jia, G., Springer, N.M., Vance, C.P., Stupar, R.M., 2011. The composition and origins of genomic variation among individuals of the soybean reference cultivar Williams 82. Plant Physiol. 155, 645-55.

Hollox, E.J., Huffmeier, U., Zeeuwen, P.L., Palla, R., Lascorz, J., Rodijk-Olthuis, D., van de Kerkhof, P.C., Traupe, H., de Jongh, G., den Heijer, M., Reis, A., Armour, J.A., Schalkwijk, J., 2008. Psoriasis is associated with increased beta-defensin genomic copy number. Nat Genet. 40, 23-5.

Jang, J.S., Simon, V.A., Feddersen, R.M., Rakhshan, F., Schultz, D.A., Zschunke, M.A., Lingle, W.L., Kolbert, C.P., Jen, J., 2011. Quantitative miRNA expression analysis using fluidigm microfluidics dynamic arrays. BMC Genomics 12, 144. 
441 Kim, W.-J., Jung, H., Gaffney, P.M., 2011. Development of type I genetic markers from

442

443

444

445

446

447

448

449

450

451

452

453

454

455

456

457

458

459

460

461 expressed sequence tags in highly polymorphic species. Mar. Biotechnol. (NY). 13, 12732.

Linzmeier, R.M., Ganz, T., 2005. Human defensin gene copy number polymorphisms: comprehensive analysis of independent variation in alpha- and beta-defensin regions at 8p22-p23. Genomics 86, 423-30.

Livak, K.J., Schmittgen, T.D., 2001. Analysis of relative gene expression data using real-time quantitative PCR and the 2(-Delta Delta C(T)) Method. Methods 25, 402-8.

Marroni, F., Pinosio, S., Morgante, M., 2014. Structural variation and genome complexity: is dispensable really dispensable? Curr. Opin. Plant Biol. 18, 31-6.

O’Leary, N.A., Gross, P.S., 2006. Genomic structure and transcriptional regulation of the penaeidin gene family from Litopenaeus vannamei. Gene 371, 75-83.

Reece, K.S., Ribeiro, W.L., Gaffney, P.M., Carnegie, R.B., Allen, S.K., 2004. Microsatellite marker development and analysis in the eastern oyster (Crassostrea virginica): confirmation of null alleles and non-Mendelian segregation ratios. J. Hered. 95, 346-52.

Rosa, R.D., de Lorgeril, J., Tailliez, P., Bruno, R., Piquemal, D., Bachère, E., 2012. A hemocyte gene expression signature correlated with predictive capacity of oysters to survive Vibrio infections. BMC Genomics 13, 252.

Rosa, R.D., Santini, A., Fievet, J., Bulet, P., Destoumieux-Garzón, D., Bachère, E., 2011. Big defensins, a diverse family of antimicrobial peptides that follows different patterns of expression in hemocytes of the oyster Crassostrea gigas. PLoS One 6, e25594. 
462

463

464

465

466

467

468

469

470

471

472

473

474

475

476

477

478

479

480

481

482

Rosa, R.D., Vergnes, A., de Lorgeril, J., Goncalves, P., Perazzolo, L.M., Sauné, L., Romestand, B., Fievet, J., Gueguen, Y., Bachère, E., Destoumieux-Garzón, D., 2013. Functional divergence in shrimp anti-lipopolysaccharide factors (ALFs): from recognition of cell wall components to antimicrobial activity. PLoS One 8, e67937.

Saavedra, C., Bachère, E., 2006. Bivalve genomics. Aquaculture 256, 1-14.

Sauvage, C., Bierne, N., Lapègue, S., Boudry, P., 2007. Single Nucleotide polymorphisms and their relationship to codon usage bias in the Pacific oyster Crassostrea gigas. Gene 406, 13-22.

Schmitt, P., Gueguen, Y., Desmarais, E., Bachère, E., de Lorgeril, J., 2010. Molecular diversity of antimicrobial effectors in the oyster Crassostrea gigas. BMC Evol. Biol. 10, 23.

Schmitt, P., Rosa, R.D., Duperthuy, M., de Lorgeril, J., Bachère, E., Destoumieux-Garzón, D., 2012. The antimicrobial defense of the Pacific oyster, Crassostrea gigas. How diversity may compensate for scarcity in the regulation of resident/pathogenic microflora. Front. Microbiol. 3, 160.

Schmitt, P., Santini, A., Vergnes, A., Degremont, L., de Lorgeril, J., 2013. Sequence polymorphism and expression variability of Crassostrea gigas immune related genes discriminate two oyster lines contrasted in term of resistance to summer mortalities. PLoS One 8, e75900.

Springer, N.M., Ying, K., Fu, Y., Ji, T., Yeh, C.-T., Jia, Y., Wu, W., Richmond, T., Kitzman, J., Rosenbaum, H., Iniguez, A.L., Barbazuk, W.B., Jeddeloh, J.A., Nettleton, D., 

Schnable, P.S., 2009. Maize inbreds exhibit high levels of copy number variation (CNV) and presence/absence variation (PAV) in genome content. PLoS Genet. 5, e1000734.

Swanson-Wagner, R.A., Eichten, S.R., Kumari, S., Tiffin, P., Stein, J.C., Ware, D., Springer, N.M., 2010. Pervasive gene content variation and copy number variation in maize and its undomesticated progenitor. Genome Res. 20, 1689-99.

Tamura, K., Dudley, J., Nei, M., Kumar, S., 2007. MEGA4: Molecular Evolutionary Genetics Analysis (MEGA) software version 4.0. Mol. Biol. Evol. 24, 1596-9.

Taris, N., Lang, R.P., Camara, M.D., 2008. Sequence polymorphism can produce serious artefacts in real-time PCR assays: hard lessons from Pacific oysters. BMC Genomics 9, 234.

Taudien, S., Galgoczy, P., Huse, K., Reichwald, K., Schilhabel, M., Szafranski, K., Shimizu, A., Asakawa, S., Frankish, A., Loncarevic, I.F., Shimizu, N., Siddiqui, R., Platzer, M., 2004. Polymorphic segmental duplications at 8p23.1 challenge the determination of individual defensin gene repertoires and the assembly of a contiguous human reference sequence. BMC Genomics 5, 92.

Wang, Y., Lu, J., Chen, S., Shu, L., Palmer, R.G., Xing, G., Li, Y., Yang, S., Yu, D., Zhao, T., Gai, J., 2014. Exploration of presence/absence variation and corresponding polymorphic markers in soybean genome. J. Integr. Plant Biol. 56, 1009-19.

Zhang, G., Fang, X., Guo, X., Li, L., Luo, R., Xu, F., Yang, P., Zhang, L., Wang, X., Qi, H., Xiong, Z., Que, H., Xie, Y., Holland, P.W.H., Paps, J., Zhu, Y., Wu, F., Chen, Y., Wang, J., Peng, C., Meng, J., Yang, L., Liu, J., Wen, B., Zhang, N., Huang, Z., Zhu, Q., Feng, Y., Mount, A., Hedgecock, D., Xu, Z., Liu, Y., Domazet-Lošo, T., Du, Y., Sun, X., 
Zhang, S., Liu, B., Cheng, P., Jiang, X., Li, J., Fan, D., Wang, W., Fu, W., Wang, T.,

506

507

508

509

510

511

512

513

514

515

516

517

518

519

520

521

522

523

524

525

526

527

528 Wang, B., Zhang, J., Peng, Z., Li, Y., Li, N., Wang, J., Chen, M., He, Y., Tan, F., Song, X., Zheng, Q., Huang, R., Yang, H., Du, X., Chen, L., Yang, M., Gaffney, P.M., Wang, S., Luo, L., She, Z., Ming, Y., Huang, W., Zhang, S., Huang, B., Zhang, Y., Qu, T., Ni, P., Miao, G., Wang, J., Wang, Q., Steinberg, C.E.W., Wang, H., Li, N., Qian, L., Zhang, G., Li, Y., Yang, H., Liu, X., Wang, J., Yin, Y., Wang, J., 2012. The oyster genome reveals stress adaptation and complexity of shell formation. Nature 490, 49-54.

Zhang, L.-M., Luo, H., Liu, Z.-Q., Zhao, Y., Luo, J.-C., Hao, D.-Y., Jing, H.-C., 2014. Genome-wide patterns of large-size presence/absence variants in sorghum. J. Integr. Plant Biol. 56, 24-37.

\section{Figure captions}

Figure 1. Polymorphism in $C g$-BigDef basal mRNA expression in 163 individual oysters. Relative mRNA levels were analyzed in oysters expressing Cg-BigDef1 (81/163), Cg-BigDef2 (47/163) and Cg-BigDef3 (21/163). Relative expression data were normalized with the Cq geometric mean value of three reference genes (Cg-efl $\alpha, C g$-rpl40 and $C g$-rps6) according to the $2^{-\Delta \Delta \mathrm{Cq}}$ method.

Figure 2. The lack of detection of $C g$-BigDef expression is likely due to the lack of the corresponding gene in the oyster genome. (A) Relative mRNA abundance of the three $C g$ BigDefs in 10 individual oysters by RT-qPCR. Relative mRNA expressions were normalized with the Cq geometric mean value of three reference genes ( $C g$-efl $\alpha, C g$-rpl40 and Cg-rps6) according to the $2^{-\Delta \Delta \mathrm{Cq}}$ method. (B) Detection of $C g$-bigdef genes by conventional PCR using 
specific (Cg-bigdef1, Cg-bigdef2, Cg-bigdef3) and universal (Cg-bigdefs) primer pairs. The quality and integrity of the gDNA samples were assessed by the amplification of the Cg-efl $\alpha$ gene.

Figure 3. Each $C g$-BigDef form is encoded by a separated gene. (A) A not-to-scale representation of Cg-bigdef genes. Black lines and boxes represent introns and exons, respectively. E=exon. (B) Multiple nucleotide alignment of the three Cg-bigdef genes. Exon sequences are in bold. The canonical GT/AG splicing recognition sites are shadowed with black backgrounds and the microsatellite sequences (CTAT, CT and CA) are indicated by rectangles.

Figure 4. Nucleotide alignment of the first exon and intron sequences from alleles of $\mathrm{Cg}$ bigdef1 and Cg-bigdef2 genes from a same individual oyster. Differences (nucleotide substitutions or gaps) between the two Cg-bigdefl alleles and between $C g$-bigdefl and $C g$ bigdef 2 genes are shadowed with black and grey backgrounds, respectively. Exon sequences are in bold and the canonical GT/AG splice site junctions are indicated by rectangles.

Figure 5. Phylogeny of the oyster big defensin family. Phylogenetic trees were constructed based on (A) genomic and (B) cDNA sequences using the Neighbour-Joining method in MEGA 4. Bootstrap sampling was reiterated 1,000 times. Sequences included in these analyses were the following: (i) Cg-bigdef genes (GenBank: JF703155-JF703160, JN251122, JN251125, JN251126, JN251128, JN251131 and JN251132) and Cg-defm gene (GenBank: AM050547) as outgroup; (ii) Cg-BigDef transcripts (GenBank: JF703137-JF703154, JN251121, JN251123, JN251124, JN251127, JN251129 and JN251130) and Cg-Defh2 (GenBank: DQ400102) as outgroup. 


\section{Supplementary data}

556 Appendix A. Cq values of three reference genes (Cg-efla, Cg-rpl40 and Cg-rps6) and Cg-

557 BigDefs (Cg-BigDef1, Cg-BigDef2 and Cg-BigDef3) analyzed by a high-throughput RT558 qPCR device in 163 individual oysters. 
560 Table 1. Nucleotide sequence of primers.

\begin{tabular}{|c|c|c|}
\hline Primer name & Forward primer (5'-3') & Reverse primer (5'-3') \\
\hline \multicolumn{3}{|c|}{ Primers for RT-qPCR and gDNA amplification } \\
\hline$C g-e f l \alpha$ & GAGCGTGAACGTGGTATCAC & ACAGCACAGTCAGCCTGTGA \\
\hline Cg-rps6 & CAGAAGTGCCAGCTGACAGTC & AGAAGCAATCTCACACGGAC \\
\hline Cg-rpl40 & AATCTTGCACCGTCATGCAG & AATCAATCTCTGCTGATCTGG \\
\hline \multirow{2}{*}{ Cg-bigdef1 } & TTCGCCTGCTTCCATACTGG & GTCATGGTCACTCCTTATTC \\
\hline & TCTTTGCTGCTTTGGTGACC & GTCATGGTCACTCCTTATTC \\
\hline \multirow{3}{*}{ Cg-bigdef2 } & TCTTTGCTGCTTTGGTGACC & CATAGTTTATCCCCTCCGTC \\
\hline & TTCGCCTGCTTCCATACTGG & AATGACTGTCATGGTCAGAA \\
\hline & AGAAGAAGGTGAGACGAG & TGATCCGCACACACCAAACG \\
\hline \multirow[t]{2}{*}{ Cg-bigdef3 } & AGAAGAAGGTGAGACGAG & GGCTGATTAATCCATGCAAG \\
\hline & & $\mathrm{C}$ \\
\hline Cg-bigdefs & CAGTCTTTGCTGCTTTGGTG & TTGGCACACGAATGACTGTC \\
\hline (universal) & TTCGCCTGCTTCCATACTGG & TTGGCACACGAATGACTGTC \\
\hline \multicolumn{3}{|c|}{ Primers for molecular cloning and sequencing } \\
\hline \multirow{3}{*}{ Cg-bigdef1 } & ACGTATAGGACTATCATGGAG & GTCATGGTCACTCCTTATTC \\
\hline & TTCCTACCAAAGAATAAGGAG & CGTGGCTCAGTAATCTCTGC \\
\hline & TGTTAACGTATAGGACTATC & AATGACTGTCATGGTCAGAA \\
\hline \multirow[t]{2}{*}{ Cg-bigdef2 } & TCTTTGCTGCTTTGGTGACC & CGTGGCTCAGTAATCTCTGC \\
\hline & TGTTAACGTATAGGACTATC & CGTGGCTCAGTAATCTCTGC \\
\hline \multirow[t]{2}{*}{ Cg-bigdef3 } & GGAGAACTGTTAGTGTGCTG & GGCTGATTAATCCATGCAAG \\
\hline & & $\mathrm{C}$ \\
\hline
\end{tabular}

561

562 
Table 2. Occurrence of basal Cg-BigDef expression in the oyster population analyzed.

\begin{tabular}{lcc}
\hline Cg-BigDef & \multicolumn{2}{c}{ Occurrence (\%) } \\
\hline Cg-BigDef1 alone & 60 & $(36.8 \%)$ \\
Cg-BigDef2 alone & 31 & $(19 \%)$ \\
Cg-BigDef3 alone & 08 & $(4.9 \%)$ \\
Cg-BigDef1 and Cg-BigDef2 & 12 & $(7.4 \%)$ \\
Cg-BigDef1 and Cg-BigDef3 & 09 & $(5.5 \%)$ \\
Cg-BigDef2 and Cg-BigDef3 & 04 & $(2.5 \%)$ \\
All three Cg-BigDef forms & 0 & $(0 \%)$ \\
Absence of Cg-BigDefs & 39 & $(23.9 \%)$ \\
\hline
\end{tabular}

Total of individuals

163

Table 3. Occurrence of basal gene expression of $\mathrm{Cg}$-BigDefs according to oyster survival

568 (surviving versus non-surviving) to virulent Vibrio infections

\begin{tabular}{lrlrl}
\hline Cg-BigDef & \multicolumn{3}{c}{ Occurrence (\%) } \\
\hline & \multicolumn{2}{c}{ Surviving } & \multicolumn{2}{c}{ Non-surviving } \\
\hline Cg-BigDef1 alone & 25 & $(55.5 \%)$ & 25 & $(55.5 \%)$ \\
$C g$-BigDef2 alone & 10 & $(22.2 \%)$ & 12 & $(26.6 \%)$ \\
$C g$-BigDef3 alone & 04 & $(8.8 \%)$ & 02 & $(4.4 \%)$ \\
Cg-BigDef1 and Cg-BigDef2 & 27 & $(60.0 \%)$ & 04 & $(8.8 \%)$ \\
$C g$-BigDef1 and Cg-BigDef3 & 06 & $(13.3 \%)$ & 31 & $(68.8 \%)$ \\
Cg-BigDef2 and Cg-BigDef3 & 0 & $(0 \%)$ & 0 & $(0 \%)$ \\
All three Cg-BigDef forms & 0 & $(0 \%)$ & 0 & $(0 \%)$ \\
Absence of Cg-BigDefs & 12 & $(26.6 \%)$ & 10 & $(22.2 \%)$ \\
\hline Total of individuals & $\mathbf{4 5}$ & & $\mathbf{4 5}$ &
\end{tabular}


Figure 1

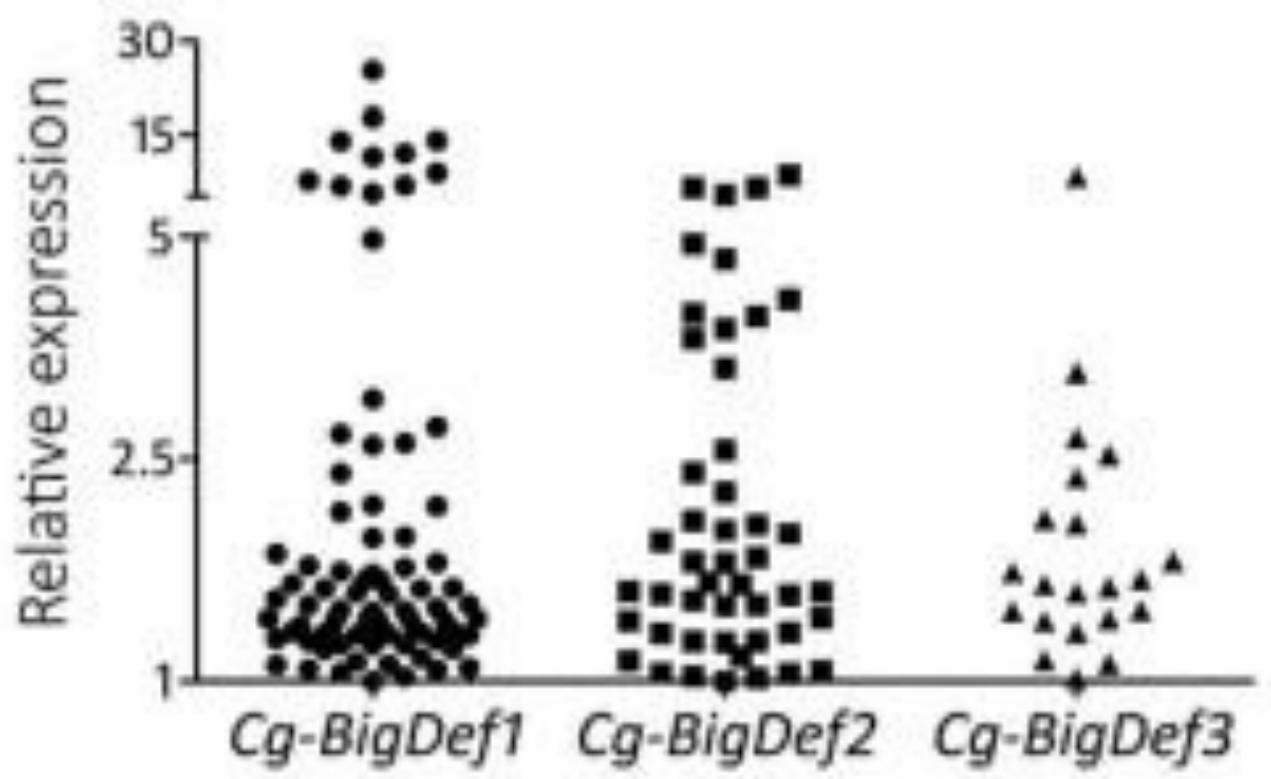

Figure 2

A

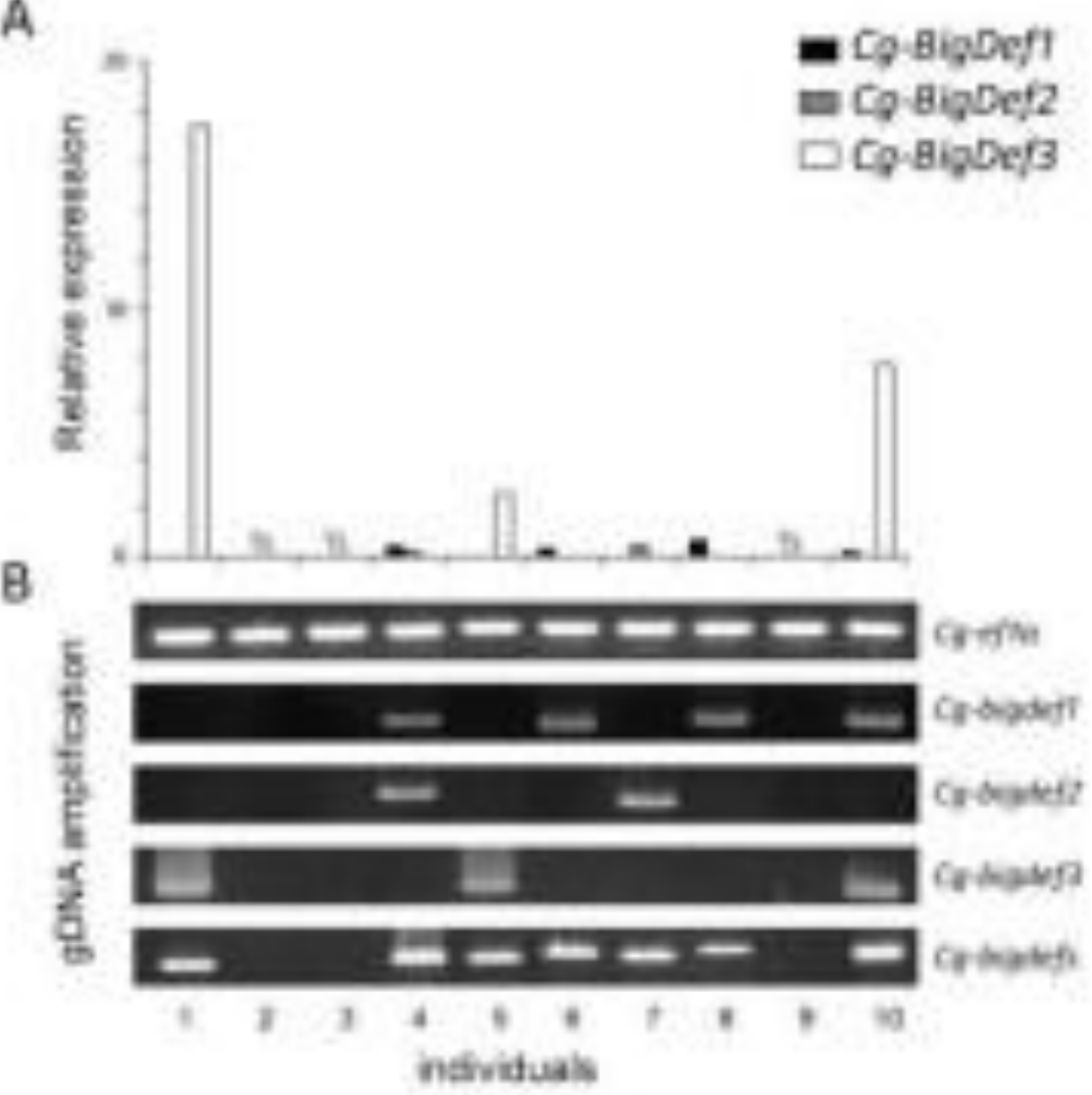


Figure 3

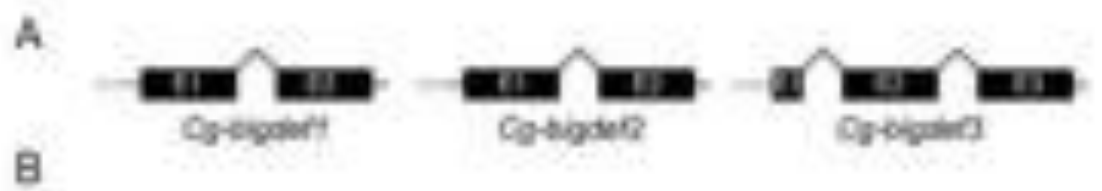

日

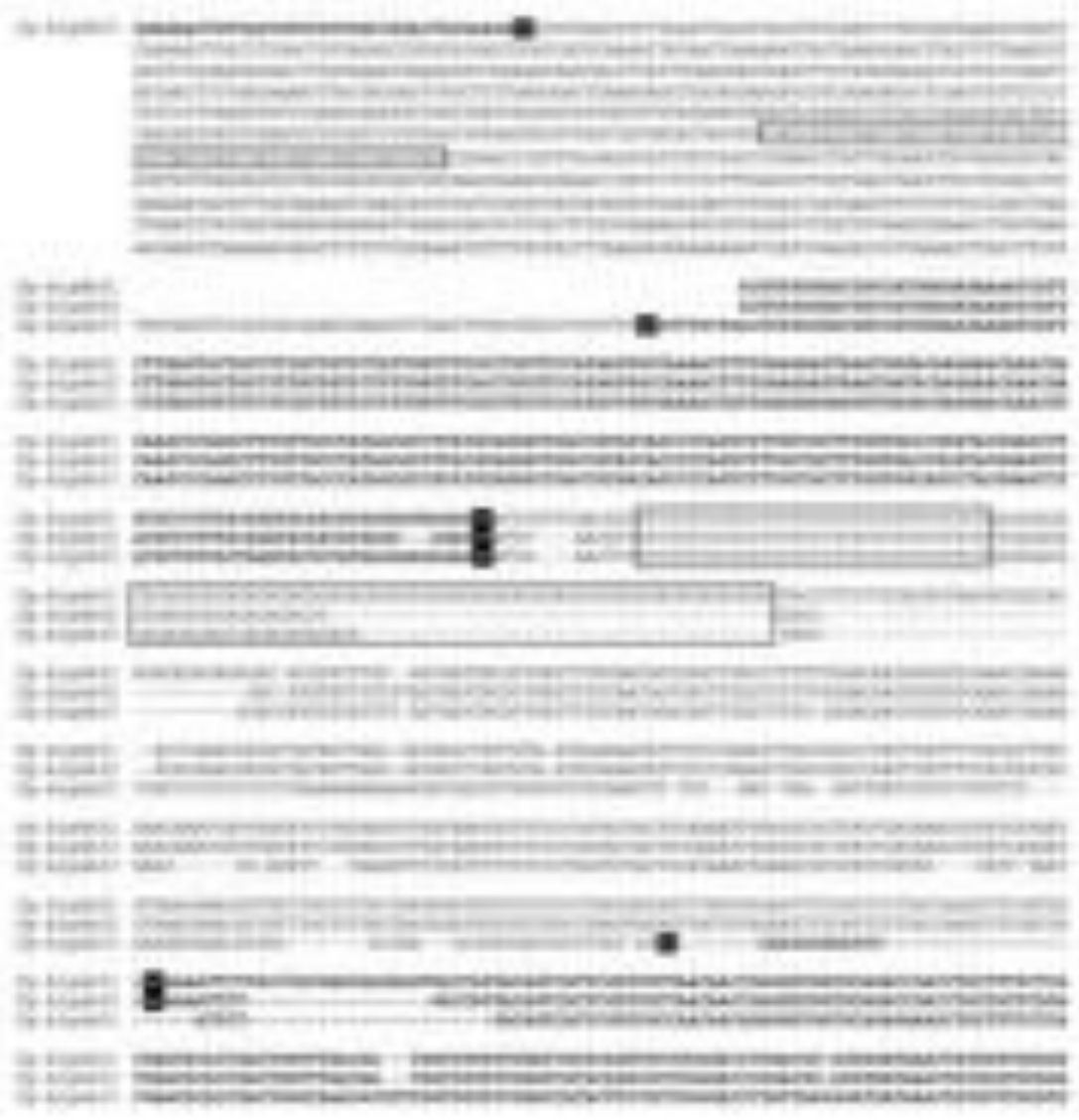


Figure 4

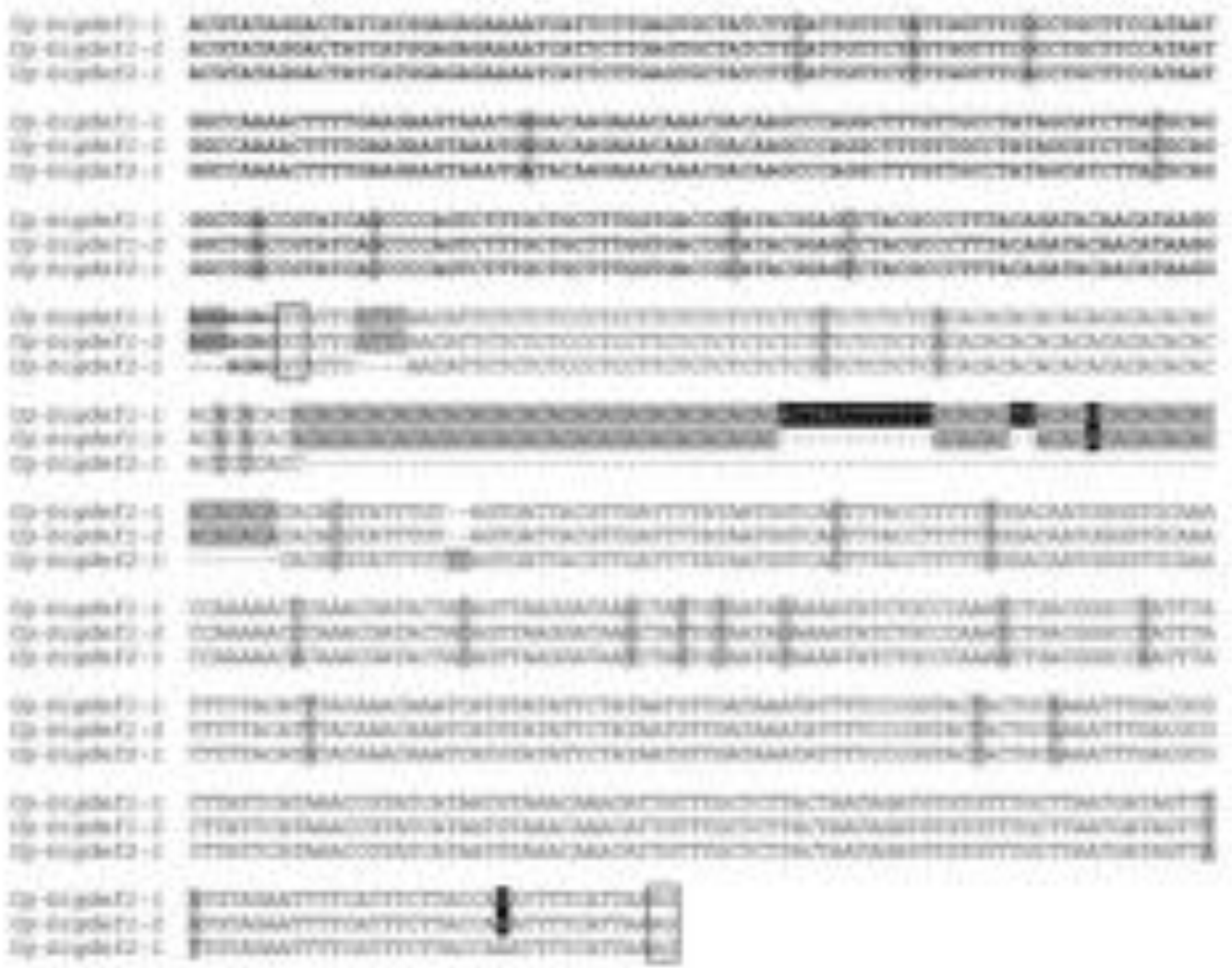


Figure 5

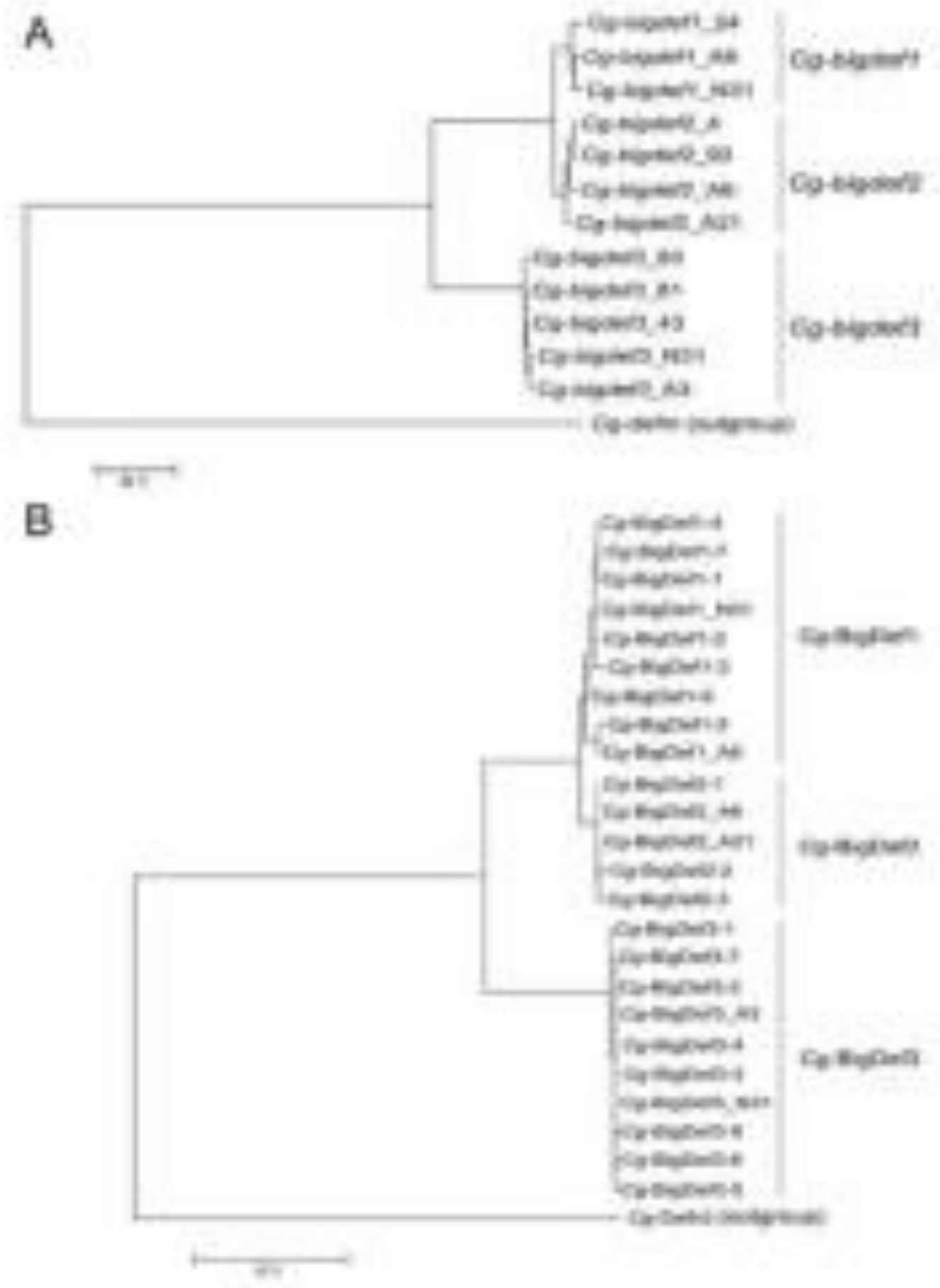

